\title{
Avaliação de Bis(4-Metilfenilditiocarbimato)Zincato(II) de Tetrabutilamônio como Acelerador em Composições de Borracha Natural
}

\author{
Roberta M. Mariano, Leila L. Y. Visconte \\ Instituto de Macromoléculas Professora Eloísa Mano, UFRJ \\ Marcelo R. L. Oliveira, Mayura M. M. Rubinger \\ Departamento de Química, Universidade Federal de Viçosa
}

Resumo: Neste trabalho a substância ZNIBU [bis(4-metilfenilditiocarbimato)zincato(II) de tetrabutilamônio] foi usada em formulações de borracha natural (NR) e o seu efeito como acelerador de vulcanização foi investigado. As composições, vulcanizadas com a substância em questão, foram submetidas a testes mecânicos e os resultados comparados com os de outras composições vulcanizadas com os aceleradores comerciais CBS (N-ciclohexil-2-benzotiazol-2-sulfenamida), TMTD (dissulfeto de tetrametiltiuram) e MBTS (dissulfeto de benzotiazol). Propriedades como dureza, resiliência e densidade foram avaliadas em presença ou não da carga negro de fumo.

Palavras-chave: Vulcanização, aceleradores, borracha natural (NR), ditiocarbimatos.

\section{Evaluation of Tetrabutyl Ammonium Bis(4-Methylphenyldithiocarbimato)Zincate(II) as Accelerator in Natural Rubber (NR) Compounds}

\begin{abstract}
The acceleration potential of ZNIBU [tetrabutyl ammonium bis(4-methylphenyldithiocarbimatozincate(II))] in the vulcanization process of natural rubber compounds was investigated. The vulcanized compounds were tested for hardness, resilience and density and compared with those vulcanized with commercial accelerators such as CBS (N-cyclohexyl-2benzothiazolesulphenamide), TMTD (tetramethyl thiuram disulphide) and MBTS (dibenzothiazole disulphide). The new accelerator tested was found to be too slow for a commercial application, but its properties were similar to those of other accelerators.
\end{abstract}

Keywords: Vulcanization, accelerators, natural rubber (NR), dithiocarbimates.

\section{Introdução}

A primeira tentativa de aumentar a velocidade da reação de vulcanização dos compostos elastoméricos surgiu com a utilização dos óxidos metálicos de chumbo, cálcio e magnésio. Entretanto, este aumento mostrou ser insuficiente para aplicações comerciais até que, em 1906, Oenslager ${ }^{[1]}$ descobriu os aceleradores orgânicos com a síntese da anilina a qual, por ser elevadamente tóxica, passou a ter o seu uso proibido na preparação de produtos de borracha. Depois disso, o uso de diversos compostos como o 2-mercaptobenzotiazol (MBT), reportado por Bedford e Sebrell ${ }^{[1]}$ e por Bruni e Romani ${ }^{[1]}$ em 1921, as guanidinas, as aldeídoaminas e os ditiocarbamatos, entre outros, tornou-se comum. Hoje, com a diversidade de substâncias disponíveis no mercado, o desenvolvimento de novos aceleradores já não suscita tanto interesse.

De acordo com sua atividade em relação à velocidade de vulcanização, o acelerador pode ser classificado como lento, médio, rápido ou muito rápido. A escolha do acelerador mais adequado a uma composição de borracha deve levar em conta, além da sua eficiência, fatores como tempo de scorch ou pré-cura, que é o tempo de segurança durante o qual não ocorre formação significativa de ligações cruzadas. A existência de aceleradores que apresentem diferentes valores de velocidade de cura e tempo de pré-cura é de extrema utilidade industrial.

Com os aceleradores disponíveis no mercado, uma prática bastante empregada é o uso de mistura de aceleradores em composições elastoméricas, com o objetivo de suprir os requisitos necessários à vulcanização que não podem ser atendidos por apenas um composto.

Em virtude das crescentes preocupações sociais e ambientais, muito dos aceleradores atualmente em uso, particularmente os geradores de nitrosaminas, tendem a ser substituídos por outros que não causem problemas à saúde e ao meio ambiente.

Autor para correspondência: Roberta M. Mariano, Instituto de Macromoléculas Professora Eloisa Mano, UFRJ, Caixa Postal 68525, CEP: 21970-170, 
As nitrosaminas são formadas por reações envolvendo aminas secundárias. Aceleradores como MBS (2-morfolinotiobenzotiazol) e TMTD são altamente tóxicos e formadores de nitrosaminas. Experimentos com animais demonstraram que as nitrosaminas são potentes carcinogênicos. O interesse pelas propriedades cancerígenas das nitrosaminas começou em 1956 com estudos sobre a N-nitrosodimetilamina (NDMA). Foi demonstrado que este composto provoca necrose centrilobular hemorrágica hepática em ratos, camundongos, coelhos e cães. Mais recentemente, registraram-se em alguns países casos de envenenamento agudo por essa nitrosamina, resultando em necrose hepática massiva, por alterações no DNA das células hepáticas ${ }^{[2]}$.

As nitrosaminas podem afetar uma grande variedade de órgãos dependendo de sua estrutura e das espécies animais usadas nos estudos. Tumores do fígado, esôfago, pulmões, mucosa nasal, bexiga, língua e estômago são frequientemente induzidos por nitrosaminas nos ratos ${ }^{[2]}$.

A N-nitrosodimetilamina é freqüentemente encontrada em alimentos ${ }^{[3,4]}$ especialmente os industrializados tais como presunto, salsicha, salame e outros derivados da carne. Quando esses alimentos são consumidos continuamente, podem provocar câncer e mutações genéticas. O perigo nos enlatados está também na forma de comercialização, pois a estocagem em ambiente fechado favorece as reações que geram nitrosaminas ${ }^{[5]}$. Nitrosaminas são formadas nos alimentos pela reação das proteínas da carne com os nitritos, sais usados como conservantes.

Os compostos $\mathrm{N}$-nitroso podem ser formados pela interação de um agente nitrosante derivado de sais de nitrato ou de óxidos de nitrogênio, com uma substância que contenha um grupo amino secundário. No processo de vulcanização da borracha, são formados em altas temperaturas, quando são usados aceleradores contendo nitrogênio como tiurans, ditiocarbamatos e derivados da morfolina. São bastante estáveis podendo permanecer por muito tempo no interior do artefato, sendo lentamente liberados para a atmosfera. Ambientes fechados, com má ventilação, como armazéns, são muito propícios à formação de nitrosaminas, devido à degradação lenta dos produtos, e por isso devem ser evitados como locais de estocagem ${ }^{[6]}$.

Neste trabalho, o composto bis(4-metilfenilditiocarbimato)zincato(II) de tetrabutilamônio, ZNIBU, foi investigado quanto ao seu potencial de aceleração da vulcanização em uma composição padrão de borracha natural (NR ${ }^{[7]}$. O ZNIBU é um sal composto por dois cátions tetrabutilamônio e um complexo aniônico de zinco com 4-metilfenilsulfonilditiocarbimato, conforme a estrutura apresentada na Figura 1.

O ZNIBU não apresenta em sua parte aniônica átomos de nitrogênio ligados a grupos alquila ou arila. Sua parte catiônica formada pelo íon tetrabutilamônio também não favorece reações de nitrosação. Portanto esse composto não pertence à classe dos formadores de nitrosaminas secundárias ${ }^{[7]}$.

A ação aceleradora do ZNIBU foi comparada à dos aceleradores comerciais CBS (N-ciclohexil-2-benzotiazol-2-sulfenamida), TMTD (dissulfeto de tetrametiltiuram) e MBTS (dissulfeto de benzotiazol), por meio de dados reométricos. As propriedades de dureza, resiliência e densidade foram também estudadas.

\section{Experimental}

\section{Material e métodos}

As composições de borracha natural foram preparadas segundo as normas ASTM D 3184-80 $0^{[8]}$ e ASTM D 3192-85 $5^{[9]}$ para as composições sem carga e reforçadas com negro de fumo, respectivamente. A formulação utilizada foi (em phr): borracha natural (NR) (100); óxido de zinco (3,5); negro de fumo (0 ou 20); ácido esteárico (2,5); aminox (antioxidante: produto da reação, a baixa temperatura, entre difenilamina e acetona) $(2,0)$; acelerador $(0,8)$ e enxofre $(2,5)$.

Os ingredientes, utilizados como recebidos, foram misturados em um misturador de rolos, marca Berstorff, com razão de fricção de 1:1,25 e velocidade dos rolos 24 (anterior) e 30 (posterior) rpm, durante cerca de 25 minutos, a temperatura ambiente, seguindo a ordem de adição dos ingredientes descrita nas normas.

As propriedades reométricas foram obtidas em reômetro de disco oscilatório, da TI 100 Instruments, a $150{ }^{\circ} \mathrm{C}$, arco $1^{\circ}$, segundo a norma ASTM D 2084-81 ${ }^{[10]}$.

Para avaliação das propriedades físico-mecânicas foram realizadas medidas de dureza (shore A) (ASTM D 2240-86) ${ }^{[11]}$, resiliência (ASTM D 2632-88) ${ }^{[12]}$ e densidade relativa (ASTM D 297-81 ${ }^{[13]}$ dos vulcanizados.

\section{Determinação das propriedades reométricas}

As composições de borracha natural, com e sem carga, foram submetidas ao reômetro de disco oscilatório durante 1 hora. Foram determinados, a partir das curvas reométricas, o torque mínimo $\left(\mathrm{M}_{\mathrm{L}}\right)$, torque máximo $\left(\mathrm{M}_{\mathrm{H}}\right)$, tempo de pré-cura $\left(\mathrm{t}_{\mathrm{S} 1}\right)$ ou scorch, índice de velocidade de cura (CRI), calculado segundo a norma ASTM D 2084-81 $1^{[10]}$, e o tempo ótimo de vulcanização $\left(\mathrm{t}_{90}\right)$, ou seja, o tempo necessário para formação de $90 \%$ das ligações cruzadas na composição.

\section{Determinação de propriedades mecânicas}

Para o ensaio de dureza foi utilizado o Durômetro Shore A e, para o ensaio de resiliência, utilizou-se o Resiliômetro Bareiss D 89610.

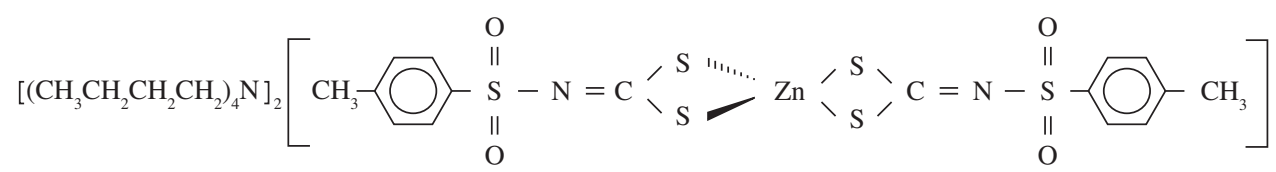

Figura 1. Estrutura do ZNIBU. 
A densidade relativa foi medida em etanol, a $23{ }^{\circ} \mathrm{C}$, e calculada segundo a norma citada.

\section{Resultados e Discussão}

As curvas reométricas de todas as composições, com e sem carga, são apresentadas na Figura 2, para todos os aceleradores utilizados neste estudo.

Na Figura 2 pode ser observado que as composições com ZNIBU apresentam tempo de pré-cura muito curto, praticamente inexistente e que, durante o experimento o valor de torque máximo somente é estabilizado no final da corrida, tanto em ausência como em presença de negro de fumo. Em presença dos outros aceleradores, é possível observar a tendência da NR à reversão, uma vez que o torque máximo, uma vez alcançado, começa a diminuir com a continuação do aquecimento.

Os parâmetros reométricos, obtidos a partir das curvas da Figura 2, são apresentados nas Tabelas 1 e 2.

De acordo com a Tabela 1, observa-se que, entre os aceleradores comerciais, o TMTD fornece um dos menores tempos de scorch e o menor valor de $\mathrm{t}_{90}$. Ele é classificado como um acelerador super rápido. Já o CBS, considerado um

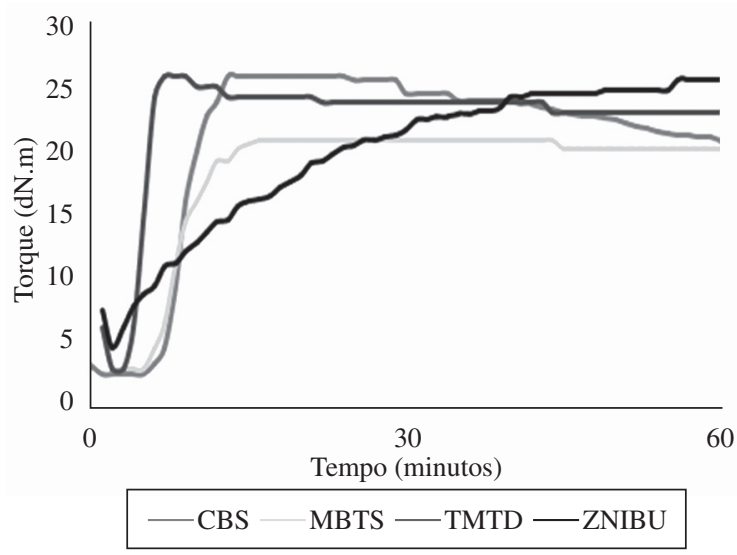

(a)

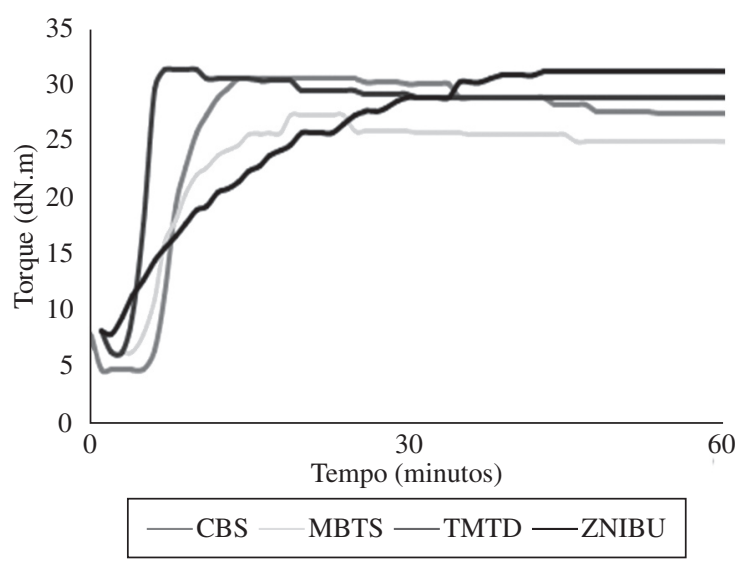

(b)

Figura 2. Curvas reométricas das composições de NR. a) Sem carga; e b) com carga. acelerador rápido, apresenta tempos de pré-cura relativamente longos; no entanto a vulcanização, uma vez iniciada, se dá rapidamente. Quando comparamos o ZNIBU aos demais aceleradores, suas composições são as que demoram mais a vulcanizar, com os maiores valores de $\mathrm{t}_{90}$, e os menores valores de scorch. Isto pode ser atribuído à possível liberação de $\mathrm{ZnS}$. De acordo com Shumane e colaboradore ${ }^{[14]}$, sistemas que usam aceleradores que liberam $\mathrm{ZnS}$ são muito lentos.

$\mathrm{O}$ processo de vulcanização envolvendo o sistema enxofre/aceleradores se passa por meio de mecanismo complexo, não totalmente elucidado, e ainda motivo de controvérsias, como é apresentado em trabalho de Kruger e colaboradores ${ }^{[15]}$. No trabalho destes pesquisadores, um sistema de vulcanização composto por NR/enxofre/ZnO e um acelerador de zinco, o dimetilditiocarbamato de zinco (ZDMC) ${ }^{[16]}$, foi utilizado em reações de vulcanização realizadas a 100 e $140{ }^{\circ} \mathrm{C}$, em diversos tempos de cura. De uma forma geral, os autores concluem que a formação de $\mathrm{ZnS}$ é característica dos sistemas formados por NR/enxofre/ZDMC/ZnO.

O torque mínimo $\left(\mathrm{M}_{\mathrm{L}}\right)$ está relacionado com a viscosidade da composição não vulcanizada e, em consequiência, com a processabilidade das composições. A Tabela 1 mostra que as composições com ZNIBU são as que apresentam os maiores valores de torque mínimo, sendo então as de mais difícil processamento. As composições com negro de fumo possuem maior viscosidade e por isso, os valores de $\mathrm{M}_{\mathrm{L}}$ são maiores que as composições sem carga. Este fato está relacionado com o caráter reforçante desta carga. O reforço é resultado do desenvolvimento de um certo grau de interação entre a carga e a matriz elastomérica, o que leva a uma restrição da mobilidade do componente polimérico, aumentando a viscosidade.

Em relação ao torque máximo $\left(\mathrm{M}_{\mathrm{H}}\right)$, observa-se que ocorre também um aumento nesses valores com a adição de carga nas composições. Este parâmetro está associado ao grau de vulcanização, isto é, ao número de ligações cruzadas formadas durante a vulcanização. $\mathrm{O}$ caráter reforçante do negro de fumo é resultado do desenvolvimento de interações entre a matriz elastomérica e a carga. Esses pontos de interação, por restringirem os movimentos moleculares das cadeias poliméricas, são responsáveis pelo aumento no torque, embora não sejam pontos de reticulação verdadeiros. Observa-se que os valores de $M_{L}$ e $M_{H}$ das composições com ZNIBU são semelhantes aos dos aceleradores usados como referência.

A Tabela 2 mostra que as composições sofrem um aumento significativo no índice de velocidade de cura (CRI) com a adição de carga, nos casos de TMTD e do ZNIBU, contrariamente ao que acontece para o CBS e o MBTS.

As composições vulcanizadas com ZNIBU apresentam valores muito baixos de CRI. Conforme a expressão utilizada para o cálculo deste parâmetro reométrico ${ }^{[10]}$, quanto menor o índice de velocidade de cura, maior a diferença entre os tempos de vulcanização e de pré-vulcanização ou scorch, enfatizando o caráter lento deste acelerador em relação à vulcanização da NR. 
Tabela 1. Dados reométricos das composições de NR, obtidos a $150^{\circ} \mathrm{C}$.

\begin{tabular}{|c|c|c|c|c|c|}
\hline Acelerador & Carga (phr) & $\begin{array}{c}\text { Torque mínimo } \\
\left(M_{1}\right)(d N . m)\end{array}$ & $\begin{array}{c}\text { Torque máximo } \\
\left(\mathrm{M}_{\mathrm{H}}\right)(\mathrm{dN} . \mathrm{m})\end{array}$ & $\begin{array}{c}\text { Tempo de pré-cura } \\
\left(\mathrm{ts}_{1}\right)(\mathrm{min})\end{array}$ & $\begin{array}{c}\text { Tempo ótimo de cura } \\
\left(t_{90}\right)(\mathrm{min})\end{array}$ \\
\hline \multirow[t]{2}{*}{ CBS } & - & 2,82 & 25,51 & 5,98 & 11,65 \\
\hline & 20 & 4,29 & 30,59 & 5,4 & 11,4 \\
\hline \multirow[t]{2}{*}{ TMTD } & - & 3,05 & 25,51 & 2,4 & 4,8 \\
\hline & 20 & 5,31 & 29,12 & 2,21 & 4,37 \\
\hline \multirow[t]{2}{*}{ MBTS } & - & 3,39 & 21,34 & 4,8 & 12,0 \\
\hline & 20 & 6,10 & 26,41 & 3,6 & 12,0 \\
\hline \multirow[t]{2}{*}{ ZNIBU } & - & 4,85 & 25,29 & 1,8 & 35,4 \\
\hline & 20 & 6,66 & 31,72 & 1,2 & 29,4 \\
\hline
\end{tabular}

Tabela 2. Índice de velocidade de cura (CRI) e variação de torque das composições de NR vulcanizadas a $150^{\circ} \mathrm{C}$.

\begin{tabular}{cccc}
\hline Acelerador & Carga $(\mathbf{p h r})$ & $\mathbf{C R I}(\mathbf{m i n})$ & $\Delta \mathbf{M}\left(\mathbf{M}_{\mathbf{H}}-\mathbf{M}_{\mathbf{L}}\right)(\mathbf{d N . m})$ \\
\hline CBS & - & 17,64 & 22,69 \\
& 20 & 16,67 & 26,30 \\
TMTD & - & 41,67 & 22,46 \\
& 20 & 46,30 & 23,81 \\
\multirow{2}{*}{ MBTS } & - & 13,89 & 17,95 \\
& 20 & 11,90 & 20,31 \\
ZNIBU & - & 2,98 & 20,44 \\
& 20 & 3,55 & 25,06 \\
\hline
\end{tabular}

Os valores de $\Delta \mathrm{M}$, diferença entre os torque máximo e mínimo, representam o aumento de torque resultante das ligações cruzadas formadas durante a vulcanização. Observase na Tabela 2 que, nas composições tipo goma pura, o valor fornecido pelo ZNIBU é superior ao do MBTS, mas não muito abaixo daqueles fornecidos pelo CBS e TMTD. Já em presença da carga, CBS e ZNIBU foram os aceleradores que apresentaram os maiores valores de $\Delta \mathrm{M}$, bastante próximos um do outro. Pode-se inferir, então que, apesar de lento, o ZNIBU fornece vulcanizados com níveis de reticulação comparáveis aos obtidos com os aceleradores comerciais.

Foram realizadas medidas de dureza (shore A), resiliência e densidade nas composições estudadas, a fim de avaliar o efeito do ZNIBU sobre essas propriedades. Os resultados estão apresentados nas Figuras 3, 4 e 5. Com relação à dureza, pode-se observar que as composições do tipo goma pura (sem carga) apresentam valores bem semelhantes, independentemente do acelerador utilizado, o mesmo acontecendo para as composições com carga.

A adição de cargas nas composições resulta em perda das propriedades elásticas, como a resiliência, quando comparado às propriedades de gomas puras. É o que mostra a Figura 4. No entanto, as composições com ZNIBU foram as que apresentaram a menor perda da propriedade com a adição do negro de fumo. Esse acelerador resultou em composições com os maiores valores de resiliência, tanto em presença como em ausência de carga.

Os resultados de densidade dos materiais podem ser vistos na Figura 5. Entre as gomas puras, aquela acelerada com o ZNIBU apresenta um dos menores valores. A adição de carga nas misturas provocou aumento da densidade em todos

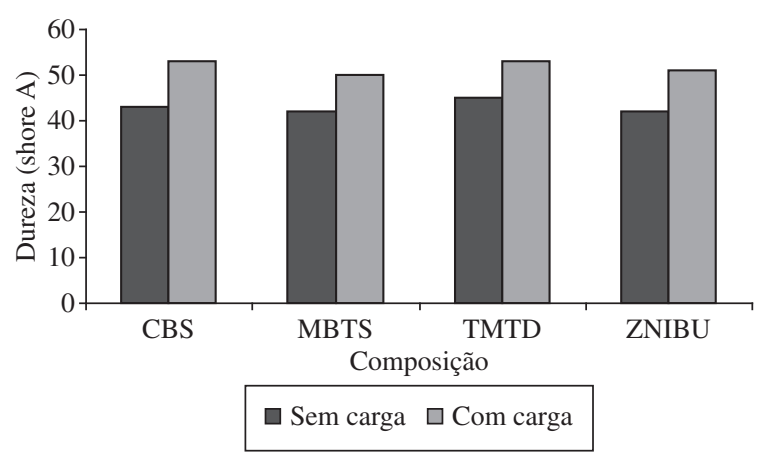

Figura 3. Resultados de dureza (shore A) das composições de NR.

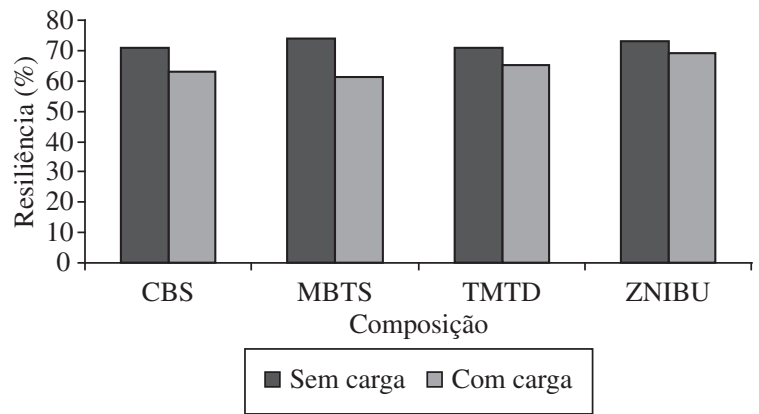

Figura 4. Resultados de resiliência das composições de NR.

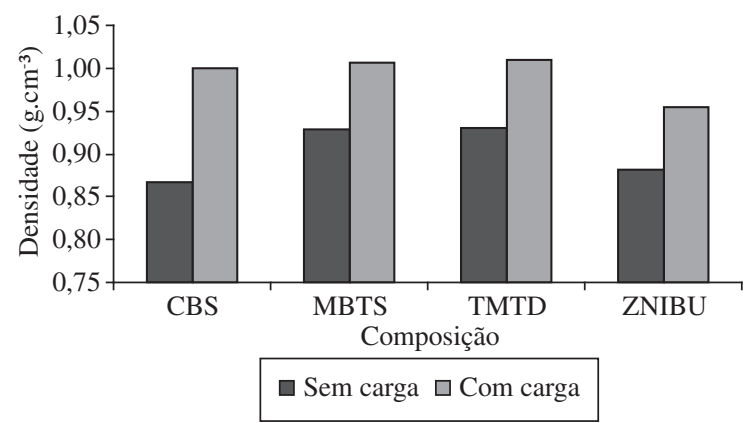

Figura 5. Valores de densidade das composições de NR.

os casos. No entanto, comparando-se as misturas, observa-se que a composição com ZNIBU apresentou o menor valor.

\section{Conclusões}

Pelos resultados reométricos apresentados, pode-se classificar o ZNIBU como um acelerador de ação muito lenta. 
Quando parte de uma formulação de borracha natural, este composto forneceu o maior valor de $t_{90}$ dentre todos os aceleradores estudados, maior ainda que o MBTS, que também é considerado um acelerador lento. $\mathrm{O}$ tempo de pré-cura fornecido pelo ZNIBU, no entanto, é muito curto.

Os resultados de dureza, resiliência e densidade, obtidos para as composições com ZNIBU, mostraram-se muito semelhantes aos dos aceleradores comerciais usados para comparação dos dados. Pode-se, a partir daí, concluir que a nova substância pode ser usada como um acelerador comercial. $\mathrm{O}$ uso de ZNIBU torna-se especialmente atraente quando se considera que este composto, diferentemente dos aceleradores comerciais, não é formador de nitrosaminas. As deficiências apresentadas podem ser sanadas pela combinação deste composto com outros aceleradores, de modo a se obterem tempos ótimos de vulcanização e de scorch, adequados a cada finalidade.

\section{Agradecimentos}

Os autores agradecem ao Conselho Nacional de Desenvolvimento Científico e Tecnológico (CNPq) pelo apoio financeiro e à Sociedade MICHELIN de Participações, Indústria e Comércio Ltda, pelo suprimento de borracha natural.

\section{Referências Bibliográficas}

1. Barlow, F. W. - "Rubber Compounding - Principles, materials and techniques", Marcel Dekker, INC. New Yord and Basel, Stow, Ohio (1988).

2. Remião F. - "Formação endógena das nitrosaminas", 2003/2004. Disponível em: http: //www.ff.up.pt/toxicologia/monografias/ano0304/Nitrosaminas/nitrosaminas.htm.

3. Bellavia V.; Natangelo M.; Fanelli R. \& Rotilio D. - J. Agriculture Food Chemistry, 48, p.1239, (2000).

4. Yurchenko S. \& Mölder U. - Food Chemistry, 96, p.325, (2006).

5. Abrantes, "Detector de nitrosaminas", Revista Ciência Hoje, 2002. Disponível em: http://www.uol.com.br/ cienciahoje/chdia/n128.htm.

6. Associação Brasileira de Tecnologia da Borracha (ABTB) - Revista Borracha Atual, 18, p.30, (1998).

7. Mariano R. M.; Oliveira M. R. L.; Rubinger M. M. M. \& Visconte L. L. Y. - Eur. Polym. J., 43, p.4706, (2007).

8. American Society for Testing and Materials (ASTM). "ASTM D 3184-80 - Rubber - Evaluation of NR (Na- tural Rubber)", USA, (1986a) (Annual Book of ASTM Standards, section 9). Disponível em: http://www. astm.org/filtrexx40.cgi?+/usr6/htdocs/astm.org/MEETINGS/meetingsindex.frm.

9. American Society for Testing and Materials (ASTM). "ASTM D 3192-85 - Carbon black in NR (Natural Rubber) - Formulation and evaluation procedures", USA, (1986b) (Annual Book of ASTM Standards, section 9). Disponível em: http://www.astm.org/filtrexx40.cgi?+/usr6/htdocs/astm.org/MEETINGS/meetingsindex.frm.

10. American Society for Testing and Materials (ASTM). "ASTM D 2084-81 - Rubber property - Vulcanization characteristics using oscillating disk cure meter", USA, (1986c) (Annual Book of ASTM Standards, section 9). Disponível em: http://www.astm.org/filtrexx40.cgi?+/usr6/htdocs/astm.org/MEETINGS/meetingsindex.frm.

11. American Society for Testing and Materials (ASTM). "ASTM D 2632-88 - Rubber property - Durometer hardness", USA, (1986d) (Annual Book of ASTM Standards, section 9). Disponível em: http://www. astm.org/filtrexx40.cgi?+/usr6/htdocs/astm.org/MEETINGS/meetingsindex.frm.

12. American Society for Testing and Materials (ASTM). "ASTM D 2240-86 - Rubber property - Resilience by vertical rebound", USA, (1986e) (Annual Book of ASTM Standards, section 9). Disponível em: http:// www.astm.org/filtrexx40.cgi?+/usr6/htdocs/astm.org/ MEETINGS/meetingsindex.frm.

13. American Society for Testing and Materials (ASTM). "ASTM D 297-81 - Rubber products - Chemical analysis", USA, (1986f) (Annual Book of ASTM Standards, section 9). Disponível em: http://www.astm.org/filtrexx40.cgi?+/usr6/htdocs/astm.org/MEETINGS/meetingsindex.frm.

14. Shumane, M.; Gradwell, M. H. S. \& McGill, W. J. - J. Appl. Polym. Sci., 86, p.1516, (2002).

15. Kruger, F. W. H. \& McGill, W. J. - J. Appl. Polym. Sci., 45, p.755, (1992).

16. Moore, C. G. \& Trego, B. R. - J. Appl. Polym. Sci., 8, p.1957, (1964).

Enviado: 03/03/08

Reenviado: $20 / 05 / 08$

Aceito: $22 / 05 / 08$ 